\title{
Strategies for Implementation of an Automated Planning System
}

\author{
Yasuo Kono ${ }^{1}$, Maurício Gonçalves Vieira Ferreira ${ }^{2}$, Francisco E. Guedes Saraiva Jr. ${ }^{3}$, Leonardo Machado Pereira ${ }^{4}$ \\ National Institute for Space Research (INPE), São José dos Campos, São Paulo, Brazil
}

\begin{abstract}
Autonomous systems for satellites planning usually utilize a knowledge base to generate flight operations plans. This knowledge base contains information that can be obtained via contributions from several experts. In a satellite control system, the knowledge base includes information about: components of the satellite, environment where the satellite operates, actions that the satellite can execute, and the effects of these actions. In planning, it is expected that the scheduling of routine actions is executed so that the satellite is operated in the best possible way. Satellite operations planning may benefit from simulators built specifically to simulate operational actions and situations before execution. This ensures that errors in planning are detected and corrected, preventing onboard failures. The objective of this paper is to show the strategies that are being applied at INPE (Brazilian National Institute for Space Research) for implementation of an automated planning system for future missions. These strategies range from the creation of a knowledge base for storage the history of all flight operations plans, up to the creation of a simulator to validate these plans, so that the satellites health is not compromised.
\end{abstract}

\section{Introduction}

A satellite control system generally uses a knowledge base that contains information obtained through contributions from various experts. The operation and control of these satellites are carried out following an action plan generated previously from autonomous planning systems.

Planning is a technique that can realize the environment, to evaluate ways to build future reference, and evaluate the process for which the plan is intended. It is part of the rational side of the actions, and a process in which actions are chosen, anticipating the results, seeking the best way to achieve a pre-defined goal. In a planning system it is expected that routine actions can be created, or that the plan can be executed in the best possible way.

A satellite, given its high cost and also the impossibility to carry out repairs, requires simulators for operational activities and operating conditions to be simulated before implementation, in order to prevent any failure that could disable the satellite, or avoid unwanted results. It is interesting to simulate, for example, the sending of a command to the satellite and verify whether this command will act on the satellite and how it will affect its working, as well as its effect under the environmental condition in which it situates.

We noticed that the utilization of a comprehensive knowledge base, which could be used for both planning and simulation has a useful application, because it allows some action to be planned on the satellite, and verify through simulation whether this action will succeed or not.

Therefore, we intend to utilize the structure defined by an object-oriented meta-model as the knowledge base, to populate with real data obtained from the Satellite Control Center - CCS, of the National Institute for Space Research - INPE (Sao Jose dos Campos / Brazil), obtained from normal routine activities or exceptional events. Through a satellite simulator, some procedures will be executed to demonstrate the possibility of integrating two works: the knowledge base, and the satellite simulator.

\footnotetext{
${ }^{1}$ Senior Computer Programmer, Satellite Tracking and Control Center (CRC), yasuo@ccs.inpe.br

${ }^{2}$ Doctor Engineer/Researcher, Satellite Tracking and Control Center (CRC), mauricio@ccs.inpe.br.

${ }^{3}$ System Analyst/Scientific Initiation Student, Satellite Tracking and Control Center (CRC), francisco@ccs.inpe.br.

${ }^{4}$ System Analyst/Scientific Initiation Student, Satellite Tracking and Control Center (CRC), leonardo@ccs.inpe.br.
} 